\title{
LOS GÉNEROS TEXTUALES Y LA PERICIA EN TRADUCCIÓN
}

\author{
Tomás Conde* \\ Universidad del País Vasco
}

\begin{abstract}
Resumen: El presente trabajo versa sobre las posibilidades que, en el ámbito de la traducción, abre tanto para la docencia como para la investigación la interconexión entre géneros textuales y pericia. Se tratan primero los conceptos de forma aislada, se comentan luego los problemas que entraña la competencia traductora $\mathrm{y}$, finalmente, se aportan diversas ideas para trabajar los géneros textuales con expertos y novatos, y viceversa. El trabajo con géneros textuales podría acelerar el desarrollo de la pericia, mediante la automatización de los procesos mentales, la facilitación de mecanismos metacognitivos y la posibilidad de que los sujetos actúen en situaciones seudorreales. Se proponen iniciativas científicas que pongan de manifiesto las diferencias sobre cómo aprovechan expertos y novatos los géneros textuales, de manera que pueda profundizarse en esta relación y justificarse con datos empíricos.
\end{abstract}

Palabras-clave: Traducción, género textual. Traductología cognitiva. Pericia. Competencia traductora.

\section{TEXTUAL GENRES AND EXPERTISE IN TRANSLATION}

\begin{abstract}
This paper deals with the implications that a potential relationship between textual genres and expertise would have both on translation teaching
\end{abstract}

\footnotetext{
* Doctor en Traducción e Interpretación por la Universidad de Granada (España). Profesor Adjunto en la Universidad del País Vasco / Euskal Herriko Unibertsitatea, Departamento de Filología Inglesa y Alemana y Traducción e Interpretación. Vitoria-Gasteiz, País Vasco, España. E-mail: tomas.conde@cogtrans.net.
} 
and research. Firstly, the concepts are defined separately, then problems inherent to translation competence are discussed, finally several ideas are introduced on how textual genres on experts and novices are to be used, and vice versa. Using textual genres might accelerate the development of the expertise levels, by automating mental processes, paving the way for metacognitive mecanisms, and allowing for the possibility that subjects work on pseudorreal situations. Scientific initiatives are proposed to shed some light on the differences among experts and novices regarding the way they use textual genres. This interconnection would be thus reinforced and justified with empirical data.

Keywords: Translation. textual genre. Cognitive translatology. Expertise. Translation competence.

\section{Introducción}

Apenas se ha analizado el concepto de género en expertos y novatos, quizás por la bisoñez del propio estudio de la pericia en Traductología. Podría pensarse que mientras que el género textual está ligado a la traducción como producto, la pericia alude a la traducción como proceso. Pero aunque esta distinción tiene sentido en absoluto impide que existan interesantes puntos de encuentro y posibilidades de investigación.

En el presente artículo se abordan primero por separado el género textual, la pericia y la competencia traductora; después, se tratan las posibles conexiones entre los dos primeros conceptos; seguidamente, se detallan algunas implicaciones didácticas de dicha relación y, por último, se ofrecen ideas concretas con vistas a iniciativas futuras.

\section{La vertiente cognitiva del género textual en GENTT}

El concepto de género textual ha sido ampliamente tratado en los Estudios de Traducción (por ejemplo, Hurtado, 2001 o Alves \& Magalhâes, 2006), aunque es el Grupo GENTT (Géneros Textuales para la Traducción) el que ha desarrollado una actividad más 
sostenida sobre dicho concepto, una «forma convencionalizada de texto que posee una función específica en la cultura en la que se inscribe y refleja un propósito del emisor previsible por parte del receptor» (García Izquierdo, 2002, 3). Una aportación valiosa de este grupo es la triple vertiente del género, a saber: formal, comunicativa y cognitiva (García Izquierdo \& Borja, 2008).

De esas tres vertientes, es la cognitiva la menos tratada hasta la fecha, con algunas excepciones. Ezpeleta $(2005,9)$ se refiere a la dimensión cognitiva como los «modos que tienen las comunidades de entender, organizar y transformar la realidad que les es propia». En un artículo posterior, parece «equiparar» los aspectos cognitivos con el hecho de que los géneros están estructurados y convencionalizados (Ezpeleta, 2007). Lo cierto es, no obstante, que los géneros son cambiantes, dinámicos e híbridos (García Izquierdo, 2005), por muy normalizados que estén, son también interpretables porque su uso es específico siempre de una situación concreta. Es decir, los géneros son fijados por la comunidad, pero son interpretados por los individuos (en este caso, por los traductores). En definitiva, este carácter relativo es uno de los aspectos que se han abordado en el estudio cognitivo del género.

Pero hay más. En relación con su dinamismo, es aconsejable concebir los géneros no sólo como productos, sino también como procesos que surgen, evolucionan y desaparecen de acuerdo a necesidades comunicativas (Bhatia, citado por García Izquierdo \& Borja, 2008). Monzó (2007) habla de la utilidad del género para la socialización secundaria del traductor, concepción que no desentona con las corrientes socio-constructivistas y los paradigmas cognitivos de segunda generación (Conde, 2014), para los cuales el significado se construye en la interacción con los otros.

Por otra parte, la vertiente cognitiva del género es útil también para la didáctica de la traducción. Por ejemplo, Montalt, Ezpeleta \& García Izquierdo (2008) consideran que los géneros sirven para la automatización de los procesos cognitivos y más concretamente, entre otras cosas, para detectar información implícita o explícita, comprender conceptos e ideas claves, identificar las relacio- 
nes conceptuales y la fuerza locucional del texto original o desarrollar los procesos de comprensión, memoria y atención. Aragonés (2008) asegura que los géneros afectan a su vez a la manera de leer e interpretar los textos y, fuera del Grupo GENTT, el investigador Carbonell i Cortés (1999) liga estos al proceso de interpretación de un texto determinado por el contexto inmediato y las circunstancias culturales anteriores.

\section{La adquisición de la competencia traductora}

Tratar el concepto de competencia traductora antes del de pericia se debe a la similitud entre estos conceptos, pero sobre todo a que ya se han publicado inspiradores estudios sobre la conexión entre géneros textuales y la adquisición de la competencia traductora (Montalt, Ezpeleta \& García de Toro, 2005; Ezpeleta, 2005; García Izquierdo, 2005; Borja, 2005; Montalt, Ezpeleta \& García-Izquierdo, 2008; García Izquierdo \& Borja, 2008).

El concepto de competencia goza de una mayor tradición que el de pericia. Para Pym (1991), la competencia en traducción es la unión de dos habilidades: la de generar una serie de soluciones en la lengua meta a un problema o término en la lengua original, y la de elegir una de las opciones de manera segura y justificada. Monzó (2008) relaciona competencia traductora profesional con aquello que nos hace traducir de manera más eficaz y eficiente; Göpferich (2009) habla de la destreza de aplicar ciertas rutinas que dan como resultado equivalentes aceptables en la lengua de destino y Bergen (2009) funde varias propuestas para presentar su propio modelo de adquisición de la competencia traductora. Al Grupo PACTE se le conoce la definición más completa y célebre de un concepto que han ido puliendo en los últimos años y cuyas características principales son las siguientes (PACTE, 2009):

- es conocimiento experto,

- básicamente procedimental, 
- incluye varias subcompetencias, y

- dispone de un componente estratégico que resulta primordial.

Pese a su amplia presencia en la bibliografía de los últimos años, los modelos de competencia traductora han recibido no pocas críticas. Las principales objeciones se ciñen, todo hay que decirlo, a los primeros modelos, basados en una estructura modular. Desde el Grupo PETRA, Muñoz (2010b) afirmaba a este respecto que es preciso tratar el concepto como un continuo y no pararnos a estudiar sus partes, sino su desarrollo. A propósito de la dicotomía competencia/ pericia, este autor (Muñoz 2010a) pone el acento en algunos puntos conflictivos de la competencia de PACTE (Muñoz 2010a):

- separa el conocimiento lingüístico del extralingüístico, pese a que las ciencias cognitivas consideran que el significado es enciclopédico;

- separa el conocimiento sobre la traducción de las competencias bilingüe y extralingüística, cuando los traductores comparten algunos rasgos con los bilingües; y la subcompetencia estratégica sigue siendo un misterio.

Togato $(2007,16)$ contesta también el primer modelo de PACTE:

«1) se centra en los textos, en el producto, y no en lo que acontece para llegar al producto; 2) postula que el significado está en los textos y no en la mente del traductor; 3) ignora la base neurobiológica de la actividad cognitiva humana, que no se desarrolla por procesos discretos y sucesivos de codificación y descodificación, como en el caso de las máquinas; 4) ignora que el proceso cognitivo humano se da en seno del entorno social; 5) incluye matices prescriptivos.»

Tras revisar otros modelos, Togato concluye que en estos existe gran disparidad entre denominaciones y conceptos básicos que se 
van adaptando según surgen nuevos datos que amplían su (siempre) mutante definición.

En cualquier caso, PACTE (2009) habla ya de años de práctica y de conocimiento experto (pericia) en un nuevo modelo que incluye todavía algunos matices discutibles. Por ejemplo, atribuyen a los expertos un rasgo («experiencia traduciendo un amplio espectro de textos») con el que cabría el debate pues, ¿dónde queda entonces la especialización? En segundo lugar, sigue siendo cuestionable que se trate de algo exclusivo de los traductores: Montalt, Ezpeleta y García-Izquierdo (2008) reconocen que su propuesta basada en el uso de géneros textuales no es válida únicamente para desarrollar la competencia traductora, sino también para potenciar otras destrezas de los mediadores culturales. Además, lo que PACTE sigue denominando competencia traductora es diferente según la dirección en que se traduce (PACTE, 2009), lo cual quiere decir que o bien hay dos tipos de competencias traductoras (¿infinitas?), o bien que, por el contrario, lo que ocurre es que los traductores tienen distintos niveles de automatización de las tareas cognitivas.

Inspirados tal vez por estas críticas, algunos investigadores prefieren directamente abandonar el concepto de competencia por el de pericia, que al menos por el momento resulta menos polémico

\section{El concepto de pericia}

Mientras que la competencia alude a lo que una persona ha de saber y aprender (conocimientos declarativos), la pericia se ocupa del manejo de mecanismos que utilizan (en este caso) los traductores expertos en el proceso de solucionar problemas y que mejora gradualmente gracias a la práctica deliberada (Shreve, 2002). Así, Togato (2007, p.22) la define como la «destreza compleja global adquirida gracias a una prolongada práctica deliberada y continua, en la que se pueden apreciar vertientes según tareas, pero no subcomponentes, en la actividad interpersonal de traducción». 
El concepto de pericia se basa pues en el desarrollo continuo de unas habilidades cognitivas que son innatas (Muñoz 2010b). En los Estudios de Traducción, que han tomado prestado este concepto de otros campos como el de la Psicología (Ericsson y Smith, citados por Muñoz, 2011; Togato, 2007), surge como una especie de continuación del concepto de competencia (Shreve, 2002) y suele marcarse el Colloquium on Translation and Cognition celebrado en Savolinna (1998) como el punto de partida para su estudio (Tirkkonen-Condit, 2002).

Desde una perspectiva cognitiva, una de las características del concepto es que admite gradaciones; es decir, la pericia es un proceso (Sirén \& Hakkarainen, 2002), un continuo en el que se pueden marcar diferentes niveles. Al mismo tiempo que van automatizando los procesos (Séguinot, 2000), los expertos devienen capaces de resolver problemas cada vez más complicados (Sirén \& Hakkarainen, 2002), si bien para la Traductología cognitiva la delimitación del problema es algo personal y, en consecuencia, lo que para unos es un problema para otros puede no serlo (Togato, 2007).

El estudio de la pericia en traducción se ha ocupado de cuestiones como el tiempo necesario para devenir expertos (Togato, 2007; Muñoz, 2011; Pérez, 2004), la diferencia entre los conceptos de experto y profesional (Sirén \& Hakkarainen, 2002) o, especialmente, la comparación entre el desempeño de los expertos $\mathrm{y}$ de los novatos.

Togato (2007) cita la autorregulación de las propias tareas como una de las características observadas en los más expertos en el campo del aprendizaje de textos. Hoffman (1996, p.85) dice que un experto es aquel que tiene «special skills or knowledge derived from extensive experience with sub-domains», mientras que Sirén \& Hakkarainen (2002) mencionan cuatro rasgos del experto que son importantes para la traducción: destreza, rapidez, mejor organización y capacidad de monitorización. Por su parte, Göpferich \& Jääskeläinen (2009) repasan las diferencias cognitivas generales que se han observado entre expertos y novatos; los expertos: 
- conocen en profundidad un dominio especializado;

- logran un alto nivel de reestructuración e interconexión de ese saber y lo convierten en conocimiento procedimental;

- son diestros en el análisis, la creatividad y otras habilidades prácticas, como la de recuperar información almacenada en su memoria a largo plazo, superar las limitaciones de su memoria de trabajo o planear la toma en consideración de muchos factores;

- automatizan su procesos mentales;

- aprenden tanto con la táctica como con la estrategia y cuentan para ello con representaciones mentales complejas de los problemas.

Para finalizar, muchos y variados estudios se han centrado en las diferencias observadas en el comportamiento de traductores expertos y traductores novatos (Alves \& Couto, 2009; Göpferich \& Jääskeläinen, 2009; PACTE, 2009; Politis, 2007; Komissarov, 1995; Künzli, 2001; Sirén \& Hakkarainen, 2002 o Tirkkonen-Condit \& Laukkanen, 1996, entre otros).

\section{Géneros y pericia}

Los géneros textuales, representantes de una concepción más formal y comunicativa, pueden y deben relacionarse con el concepto de pericia, más tratado desde el paradigma cognitivo de los Estudios de Traducción. Esta relación tiene sentido primero porque el concepto de género textual acoge también en su seno una vertiente cognitiva (Montalt, Ezpeleta \& García-Izquierdo, 2008) y segundo porque los géneros son fenómenos reconocidos por los miembros expertos de una comunidad determinada (Swales, citado por Monzó, 2007). En este sentido, Engberg (2010) critica las propuestas tradicionales de la vertiente (meramente) comunicativa del género, pues dejan de lado, por ejemplo, el sistema cognitivo de los expertos. Para el autor, las nuevas perspectivas que aporta 
el estudio de los procesos cognitivos han de variar necesariamente las concepciones actuales, que se ven afectadas por «the attitudes, basic assumptions and knowledge resources of individual experts, and on the social process of creating and recreating meaning» (Engberg, 2010, p.59).

Los géneros textuales, las relaciones entre los mismos, y las comparaciones que se puedan hacer entre los sistemas de unos países y otros suponen una ayuda inestimable para acelerar el aprendizaje sobre los dominios de conocimiento en los que están presentes. Papavassiliou (2007) afirma que es necesario preguntarse cómo se establece la relación entre texto y sistema sociocultural y cómo difieren en ello los traductores expertos y los novatos. Parece obvio que los géneros textuales, por su naturaleza, nos ofrecen una herramienta muy útil para ambas posibilidades de investigación. No obstante, tres son las razones principales por las que los géneros textuales pueden usarse para desarrollar la pericia en traducción: el estímulo de automatismos, el impulso de lo metacognitivo y la imitación de entornos seudorreales.

\subsection{Automatización}

El trabajo con géneros textuales en la traducción de textos puede impulsar la automatización de diversos procesos mentales. En García-Izquierdo \& Monzó (2002) se infiere un interés claro por que los estudiantes, mediante corpus textuales, automaticen las regularidades relacionadas con la terminología o la estructura formal de los documentos, entre otros aspectos, mientras que Borja (2004) cita la automatización de procesos como uno de los retos que habría que afrontar el campo de la traducción jurídica.

¿Son realistas estas pretensiones desde el punto de vista de la Traductología cognitiva? Los paradigmas cognitivos de segunda generación describen las actividades mentales como procesos de reconocimiento de patrones (Togato, 2007). Martín de León $(2007$, p.6) afirma que: 
«La identificación de patrones que se repiten pone en marcha procesos de automatización y creación de subrutinas, que proporcionan fluidez al proceso traslativo y dan lugar a una competencia como la del experto, que no se reduce al conocimiento inicial del esquema explícito, impuesto de arriba abajo, sino que emerge de forma flexible de las secuencias de actividad adaptadas a cada situación.»

Los géneros, en cuanto patrones típicos, podrían ayudar a automatizar tareas y liberar recursos cognitivos para la traducción, pues los procesos de resolución de problemas se benefician de los recursos cognitivos liberados por las rutinas automatizadas (Muñoz 2010b).

Los expertos cuentan probablemente con un mapa mental de géneros que les ayuda a reducir la complejidad de los documentos que traducen. Tirkkonen-Condit (2002) dice que más que obcecarse en enseñar algo que se aprende de manera natural, lo que hay que hacer es proporcionar unas condiciones para que los sujetos alcancen el nivel experto. Por ello, es necesario aprovechar los géneros textuales no solo como arsenal de conocimiento declarativo, sino también como material constitutivo de una auténtica rutina de entrenamiento: los expertos se hacen con la práctica. Coincide Togato (2007) en abogar por una rutina de entrenamiento para automatizar soluciones y utilizar material adecuado que propicie la recurrencia de patrones de traducción en determinados dominios. Uniendo ambas ideas, es evidente que los géneros suponen una herramienta nada desdeñable para trabajar y favorecer los procesos susceptibles de automatización.

\subsection{Metacognición}

Uno de los rasgos de los expertos es que dominan los procesos metacognitivos. La familiarización con los géneros textuales podría favorecer el desarrollo del conocimiento de los aprendices, 
su actividad metacognitiva y su concienciación sobre el fenómeno. Togato (2007) se refiere al desarrollo de la pericia del traductor como un proceso en el que confluyen dos actividades: por una parte, el reconocimiento de patrones y regularidades (véase §5.1) y, por otra, el aprovechamiento del apoyo externo que entra en juego cuando no son posibles dichos patrones.

Las señales y herramientas externas, situadas en el entorno social, guían la acción autocontrolada y metacognitiva y permiten reducir los problemas complejos. Los saberes enciclopédicos pueden funcionar como señales externas; de hecho, Alves \& Magalhâes (2006) demostraron que la instrucción de conocimiento declarativo en traductores novatos acarreaba beneficios en el desarrollo de la competencia en traducción. La evaluación también puede ayudar a que el estudiante tome conciencia de su propio aprendizaje, de sus aciertos y de sus fallos, como indica Kiraly (2000) a propósito de la evaluación ipsativa.

Pero al igual que el saber declarativo o la actividad evaluadora, los géneros textuales pueden considerarse pistas externas que ayudan a reducir la complejidad y a resolver el problema de la traducción, propulsando la acción inteligente y metacognitiva.

\subsection{Simulación}

Los géneros textuales representan situaciones seudorreales que contribuyen a que el aprendiz construya su conocimiento de manera comprehensiva, no aislada, sino en consonancia con los sistemas propugnados desde el conexionismo.

Llega un momento en que los sujetos dejan de beneficiarse simplemente con la práctica de una actividad y que para que esa mejora continúe son necesarios determinados tipos de experiencia relevante, esto es, de práctica deliberada (Muñoz, 2011). No basta con practicar por practicar, sino que, alcanzado un nivel, hay que trabajar teniendo en cuenta el entorno y la construcción social del significado. Muñoz (citado por Togato, 2007) afirma que, aunque 
vagos, existen límites a la interpretación de los textos entre otras cosas porque los seres humanos experimentamos una socialización parecida y partimos de una base corporal común. No es de extrañar que se vea en los géneros una manera de mejorar la efectividad profesional de los estudiantes (Monzó, 2001), habida cuenta de que, como parte de esa socialización, pueden influir en el establecimiento de dichos límites de comprensión.

Los géneros textuales, que parten de la práctica profesional y que de alguna manera reproducen e invocan ceremonias reales, aportan ese plus de realidad y significado a las prácticas de traducción de los alumnos. Habría que aprovechar, además, la existencia de géneros más extensos para llevar a cabo proyectos de traducción en grupo que imiten en la medida de lo posible el entorno profesional, en el que los distintos actores se especializan en tareas concretas: cuando se eligen géneros textuales extensos, como núcleo de determinados encargos que simulan tareas profesionales y complejas (Togato, 2007), se trabaja la práctica relevante. El trabajo colaborativo es una manera idónea de desarrollar la pericia del traductor (Kiraly, 2000), ya que los estudiantes aprenden cuáles son los roles que tendrán que asumir en su futuro profesional, mientras se ven expuestos a gran variedad de experiencias que pueden reforzarse, a su vez, con la práctica aislada de determinadas capacidades cognitivas o el uso de diarios, porfolios o actividades de autoevaluación (Muñoz, 2011).

\section{Conclusiones}

Cabe considerar la traducción de géneros textuales específicos una fuente de información valiosa sobre la manera de traducir de los expertos. Es difícil observar su proceso de traducción - han automatizado su manera de actuar- pero, como afirma Séguinot (2000), disponemos del producto de su trabajo, con el que es posible inferir esos procesos. En cualquier caso, los resultados obtenidos mediante la investigación empírica sobre la pericia deben usar- 
se para mejorar las estrategias pedagógicas (Muñoz 2007), también en su relación con los géneros textuales, como ponen de manifiesto las ideas que se han ido apuntando en el §5.

Las aplicaciones didácticas comentadas tienen una serie de consecuencias positivas para los alumnos, como la mejora de su confianza-una de las características del perito (Tirkkonen-Condit \& Laukkanen, 1996)—o de su creatividad. No hay que desdeñar lo que los géneros puedan ofrecer para dirigir el propio aprendizaje (de géneros más difíciles a géneros más complejos, de géneros más normalizados a géneros más híbridos, etcétera) e, incluso para el propio diseño curricular. Dragsted (2004) demostró que las diferencias entre expertos y novatos se neutralizaban cuando se trabajaba con textos más difíciles, lo cual lleva a pensar que el tipo de texto y su dificultad afectan al comportamiento de expertos y novatos. Parece haber, pues, una relación entre dificultad de los textos y pericia, algo que también podría estudiarse con respecto a los géneros de especialidad. Por otra parte, Göpferich \& Jääskeläinen (2009) sostienen que las mejoras en la competencia no son visibles hasta pasados dos o tres años. Esto quiere decir que la distribución de géneros textuales según grados de pericia no debería limitarse al diseño de cada asignatura, sino que más bien habría que hacerla teniendo en cuenta un espacio de tiempo mayor.

La conexión entre géneros textuales y pericia en traductores está cobrando sentido a medida que se producen avances en ambos campos de investigación. Es preciso observar cómo utilizan los géneros (y los corpus de géneros) los expertos y los novatos, para ver en qué aspectos se diferencian y, a la postre, qué se puede enseñar para salvar esas diferencias. ¿Cómo interpretan, novatos y expertos los géneros? ¿Cómo mejoraría la actuación de ambos con una formación específica en géneros? ¿Propulsa el conocimiento (metacognitivo) de los géneros la pericia de los novatos hasta acelerar su conversión en expertos? Para intentar responder estas preguntas, el presente trabajo propone el desarrollo de iniciativas científicas, basadas en enfoques cognitivos, que aprovechen la recopilación o corpus de géneros textuales (como el Corpus GENTT), así como los distintos 
estudios ya realizados sobre géneros aislados y su relación con las comunidades que los usan, esto es, con los expertos que en último término son los responsables de crearlos, fijarlos y definirlos.

\section{Referencias}

Alves, Fabio y C.M. Magalhães. "Investigando o Papel do Monitoramento Cognitivo-Discursivo e da Metareflexão na Formação de Tradutores." Cadernos de Tradução 17.1 (2006): 71-127. Web. 9 July 2013.

y Daniel Couto. "Probing the Unit of Translation in Time: Aspects of the Design and Development of a Web Application for Storing, Annotating, and Querying Translation Process Data." Across Languages and Cultures 10.2 (2009), 251-273. Print.

Aragonés, Maite. «La Contextualización de la Patente. Primera Parte.» Intercambios 12.2 (2008): 9-11. Web. 9 July 2013.

Bergen, David. „The Role of Metacognition and Cognitive Conflict in the Development of Translation Competence." Across Languages and Cultures 10:2 (2009), pp. 231-250. Print.

Borja, Anabel. „La Investigación en Traducción Jurídica. “ Panorama actual de la investigación en traducción e interpretación. Coords. Miguel Ángel García Peinado y Emilio Ortega. Granada: Atrio, 2004. pp. 415-426. Print.

. „Organización del Conocimiento para la Traducción Jurídica a través de Sistemas Expertos Basados en el Concepto de Género Textual." El Género Textual y la Traducción. Reflexiones Teóricas y Aplicaciones Pedagógicas. Ed. Isabel García Izquierdo. Berna: Peter Lang, 2005. pp. 37-67. Print. 
Carbonell i Cortés, Ovidi. Traducción y Cultura, de la Ideología al Texto. Salamanca: Salamanca Ediciones Colegio de España, 1999. Print.

Conde, Tomás. „Traduccón, Géneros Textuales y Enfoques Cognitivos. “ Hermeneus 16 (2014). Print.

Dragsted, Barbara. "Segmentation in Translation and in Translation Memory Systems. An Empirical Investigation of Cognitive Segmentation and Effects of Integrating a TM System into the Translation Process." Diss. Copenhagen Business School, 2004. Print.

Engberg, Jan. "Knowledge Construction and Legal Discourse: The Interdependence of Perspective and Visibility of Characteristics." Journal of Pragmatics 42 (2010): pp. 48-63. Web. 9 July 2013.

Ezpeleta, Pilar. "La Noción de Género en la Planificación de la Docencia de la Traducción de la Primera Lengua Extranjera." El Género Textual y la Traducción. Reflexiones Teóricas y Aplicaciones Pedagógicas. Ed. Isabel García Izquierdo. Berna: Peter Lang, 2005. pp. 135-158. Print.

. "El Informe Técnico. Estudio y Definición del Género Textual.” La Tra-

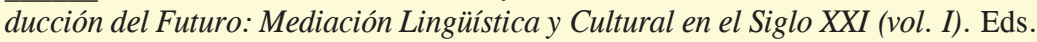
Luis Pegenaute, Janet DeCesaris y Mercè Tricás. Barcelona: Universitat Pompeu Fabra, 2007. pp. 429-438. Print.

García Izquierdo, Isabel y Esther Monzó. "Enciclopèdia Electrònica de Gèneres per a la Traducció." La Traducción Científico Técnica y la Terminología en la Sociedad de la Información. Eds. Amparo Alcina y Silvia Gamero. Castellón de la Plana: Publicacions de la Universitat Jaume I, 2002. pp. 147-154. Print.

. "El Género: Plataforma de Confluencia de Nociones Fundamentales en Didáctica de la Traducción.” Discursos 2 (2002): Lisboa: Universidade Aberta.

. "El Género y la Lengua Propia: el Español de Especialidad.” El Género Textual y la Traducción: Reflexiones Teóricas y Aplicaciones Pedagógicas. Ed. Isabel García Izquierdo. Berna: Peter Lang, 2005. pp. 117-134. Print. 
y Anabel Borja. "A Multidisciplinary Approach to Specialized Writing and Translation Using a Genre Based Multilingual Corpus of Specialized Texts." LSP \& Professional Communication, Fagsprog og Fagkommunikation, An International Journal 8.1 (2008): pp. 39-63. Print.

Göpferich, Susanne. "Towards a Model of Translation Competence and its Acquisition: the Longitudinal Study 'TransComp'." Behind the Mind: Methods, Models and Results in Translation Process Research. Eds. Susanne Göpferich, Arn Lykke Jakobsen, and Inger M. Mees I. Copenhagen: Samdundslitteratur, 2009. pp. 11-37. Print.

y Riita Jääskeläinen. „Process Research into the Development of Translation Competence: Where Are We, and Where Do We Need to Go." Across Languages and Cultures 10:2 (2009). pp. 169-191. Print.

Hoffman, Robert R. „How Can Expertise be Defined? Implications of Research From Cognitive Psychology. "Exploring Expertise. Eds. Robin Williams, Wendy Faulkner y James Fleck (Eds.), Edimburgo: University of Edinburgh Press, 1996. Web. 10 July 2013.

Hurtado, Amparo. Traducción y Traductología. Introducción a la Traductología. Madrid: Cátedra, 2009. Print.

Kiraly, Don. A Social Constructivist Approach to Translator Education. Empowerment from Theory to Practice. Manchester: St. Jerome, 2000. Print.

Komissarov, Vilen N. „Intuition in Translation.“ Target 7:2 (1995): pp. 347-354. Print.

Künzli, Alexander. „Expert versus Novices: 1'Utilisation de Sources d'Information pendant le Processus de Traduction. “ Meta 44.3 (2001): pp. 507-523. Print.

Martín de León, Celia. „Traducción y Modelos Cognitivos.“ 2007: pp. 1-6. Academia. Web. 10 July 2013.

Montalt, Vicent, Cristina García de Toro y Pilar Ezpeleta. „Elaboració de Materials Docents per a l'Ensenyament dels Gèneres Científics i Tècnics en Català, Espanyol i Anglés i la seua Traducció. " El Camí Cap a la Convergència Educa- 
tiva a Europa: una Oportunitat per a la Millora Educativa en l'UJI. Eds. Leonor Lapeña y Miguel Ángel Fortea. Castellón de la Plana: Universitat Jaume I, 2005. pp. 554-564. Print.

, Pilar Ezpeleta, and Isabel García Izquierdo. „The Acquisition of Translation Competence through Textual Genre. "Translation Journal 12:4 (2008). pp. 1-12. Print.

Monzó, Esther. „Textos Jurídics i Traduccions: Testimonis de Coneixements i Eines de Formació per al Traductor Jurídic. “ Revista de Llengua i Dret 36 (2001): pp. 23-40. Print.

. „Estudi Sincrònic i Multilingüe de Textos Jurídico-Administratius per a l'Elaboració d'un Marc d'Anàlisi Teòrico-Descriptiu. “ Diss. Universitat Jaume I, 2007. Web. 15 June 2010.

. „Derecho y Traductología en la Formación del Traductor Jurídico: una Propuesta para el Uso de Herramientas de Formación Virtual. “ Translation Journal 12:2 (2008): n. pag. Web. 10 July 2013.

Muñoz, Ricardo. „Apuntes para una Traductología Cognitiva.“ La Traducción del Futuro: Mediación Lingüística y Cultural en el Siglo XXI (vol. II). Eds. Luis Pegenaute, Janet DeCesaris y Mercè Tricás. Barcelona: Universitat Pompeu Fabra, 2007. pp. 65-74. Print.

. "Leave no Stone Unturned: On the Developement of Cognitive Translatology." Translation and Interpreting Studies 5.2 (2010a): 145-162. Print.

. "On Paradigms and Cognitive Translatology." Translation and Cognition. Eds. Gregory M. Shreve, and Erik Angelone. Amsterdam: John Benjamins, 2010b. pp. 169-187. Print.

. "Nomen Mihi Legio Est. A cognitive Approach to Natural Translation." Interpreting Naturally. Eds. María Jesús Blasco y Amparo Jiménez. Frankfurt: Peter Lang, 2011. pp. 35-66. Print. 
PACTE. "Results of the validation of the PACTE Translation Competence Model: Acceptability and Decision Making." Across Languages and Cultures 10:2 (2009): pp. 207-30. Print.

Papavassiliou, Périclès. "Traductologie et Sciences Cognitives: une Dialectique Prometteuse.” Meta 52.1 (2007): pp. 29-36. Print.

Pérez Echeverría, María del Puy. "Solución de Problemas." Psicología del Pensamiento. Coords. Mario Carretero y Mikel Asensio. Madrid: Alianza, 2004. pp. 145-192. Print.

Politis, Michel. "L'Apport de la Psychologie Cognitive à la Didactique de la Traduction." Meta 52.1 (2007): pp. 156-163. Print.

Pym, Anthony. "Translation Error Analysis and the Interface with Language Teaching." Teaching Translation and Interpreting Training Talent and Experience. Papers from the First Language International Conference. Eds. Cay Dollerup y Anne Loddegaard. John Benjamins: Amsterdam/Philadelphia, 1991. pp. 279-288. Print.

Séguinot, Candace. "Knowledge, Expertise and Theory in translation." Translation in Context. Selected papers from the EST Congress. Eds. Andrew Chesterman, Natividad Gallardo San Salvador e Yves Gambier. Granada: Benjamins, 2000. 87-104. Print.

Shreve, Gregory. "Knowing Translation: Cognitive and Experiential Aspects of Translation Expertise from the Perspective of Expertise Studies." Translation Studies: Perspectives on an Emerging Discipline. Ed. Alessandra Riccardi. Cambridge: CUP, 2002. pp. 150-171. Print.

Sirén, Seija y Kai Hakkarainen. "Expertise in Translation." Across Languages and Cultures 3.1 (2002): 71-82. Print.

Tirkkonen-Condit, Sonja. "Process Research: State of the Art and Where to go Next?" Across Languages and Cultures 3.1 (2002): 5-19. Print. 
and Johanna Laukkanen. "Evaluations - A Key Towards Understanding the Affective Dimension of Translational Decisions." Meta 41.1 (1996): pp. 4559. Print.

Togato, Giulia. "Lo que Hay que Tener. Estudio Empírico de la Pericia en Traducción.” Diss. Universidad de Granada, 2007. Print.

Recebido: 15-01-14

Aceito: 27 - 04- 14 